\title{
GROUPES DE PRESSION EN FRANCE ET AILLEURS
}

By JACQUES FREYMOND

Henry W. Ehrmann, ed, Interest Groups on Four Continents, Pittsburgh, Pa., University of Pittsburgh Press, 1958, 316 pp. $\$ 6.00$.

Jean Meynaud, Les Groupes de Pression en France (No. 95, Cahiers de la Fondation Nationale des Sciences Politiques), Paris, Armand Colin, 1958, $37 \mathrm{rpp}$.

$\mathrm{Q}$

UE l'étude des groupes de pression ait fait, au cours de ces dernières années, de réels progrès hors des Etats-Unis, et plus particulièrement en Europe, nul n'en doute. Il suffit pour s'en convaincre de lire deux ouvrages parus en 1958. Dans l'un, Jean Meynaud, professeur de science politique aux universités de Lausanne et de Genève, s'attache à l'examen des groupes de pression en France, tandis que l'autre, publié sous la direction du professeur Henry W. Ehrmann, couvre un champ plus vaste en donnant un aperçu de l'état des problèmes dans les quatre continents.

Jean Meynaud, au moment où il entreprenait cette étude, connaissait le nombre et la dimension des obstacles qu'il allait rencontrer. Il savait qu'il pénétrait sur une terre encore loin d'être défrichée et que, si bien équipé qu'il fût lui-même, les instruments de travail dont il pouvait disposer étaient singulièrement insuffisants. Tout cela il le dit dans son introduction: A "l'insuffisance de la documentation" s'ajoute "la faiblesse," "l'inexistence de la théorie politique." C'est pourquoi cet audacieux très sagement limite ses objectifs: il s'agit avant tout, dans cet ouvrage, de "faciliter l'établissement des études monographiques et la formulation des normes méthodologiques qui permettront de les dépasser." On saura gré à l'auteur de cette réserve qui, loin d'être imposée par l'ignorance, est au contraire le produit d'une longue et fructueuse recherche conduite par un esprit pénétrant. Cette étude est d'un homme admirablement informé de l'histoire de son pays, et capable aussi bien de procéder à une analyse poussée des structures sociales que d'apprécier l'influence plus subtile des jeux politiques. D'où l'articulation si claire d'un ouvrage qui, après avoir défini le phénomène de pression et circonscrit ce "groupe de pression" aux contours imprécis, donne un remarquable tableau d'ensemble des divers types d'organisation, des 
éléments de leur puissance (structures internes-techniques d'actionstatut social), des facteurs de tactique (voies d'accès-distribution des intérêts), pour terminer par un examen de la place des groupes de pression dans la vie politique, auquel il ajoute même un essai d'application des critères et des cadres de références qu'il a élaborés aux groupes internationaux.

Jean Meynaud avait voulu créer une base de départ pour d'autres études plus poussées. Il y a pleinement réussi. Car son ouvrage n'est pas seulement une excellente introduction à l'analyse de la vie politique en France. Plus encore que des renseignements de faits ou un essai de classification des divers types de groupes de pression, de leur importance relative et des modalités de leur action, il a fourni une méthode d'investigation et d'évaluation. Tous les "political scientists" qui, à l'avenir, aborderont le sujet, trouveront dans cet ouvrage le meilleur guide et tout ce qu'on peut souhaiter, c'est qu'ils y mettent autant de clarté et de sens des nuances que celui qui leur ouvre ainsi la voie. Ce livre, en effet, est marqué par la probité intellectuelle de son auteur et par une culture qu'on ne trouve pas toujours chez les spécialistes de science politique. Jean Meynaud ne prétend pas découvrir un phénomène nouveau. Il rappelle que les sociétés politiques des siècles précédents ont, elles aussi, subi l'influence de groupes de pression dont les historiens ont parfois reconstitué l'action. Loin de chercher à réserver une espèce de monopole de ce type d'étude aux "political scientists," il pousse au contraire à une collaboration interdisciplinaire, car il sait bien que rien ne serait plus faux que d'isoler une catégorie de phénomènes pour leur attribuer une influence prépondérante ou exclusive. Très justement, il dénonce la tendance actuelle à tout expliquer par le lobby érigé en mythe. "Il est toujours tentant," écrit-il, "de partir à la recherche du facteur unique d'explication d'une situation complexe: la commodité acquise grâce à l'effort de simplification l'est au détriment de la plénitude et plus simplement du réalisme de l'interprétation. Si importante soit l'étude des groupes de pression pour la compréhension des phénomènes, elle ne constitue qu'une partie de la tâche à remplir pour une analyse complète et équilibrée du système politique" (p. 347). C'est le bon sens même.

Interest Groups on Four Continents a été, on le sait probablement, préparé dans des circonstances particulières. Il s'agit d'un ouvrage collectif qui contient les rapports préparés pour la "Round Table" tenue à Pittsburgh en septembre 1957 par l'Association internationale de Science 
politique. On y trouve par conséquent, après le mémoire introductif de Henry W. Ehrmann, rapporteur général, les rapports nationaux sur l'Australie (W. A. Townsley), la Finlande (Lola Krusius-Ahrenberg), la France (Georges Lavau), l'Allemagne (Wolfgang Hirsch-Weber), la Grande-Bretagne (Samuel E. Finer), le Japon (Kiyoski Tsuji), la Suède (Gunnar Heckscher), les Etats-Unis (Samuel J. Eldersveld), la Yougoslavie (Jovan Djordjevic). Un compte-rendu des débats qui se sont déroulés à Pittsburgh constitue la dernière section du volume.

Si l'ouvrage de Jean Meynaud prétendait constituer un point de départ pour l'étude des problèmes dans un pays, celui dont Henry W. Ehrmann assume avec autorité la direction, entend présenter, par une coupe à travers les divers pays considérés, un état de la question. On pourrait penser que dans ces conditions, ce livre pèche par le manque d'unité qui caractérise les ouvrages collectifs. Ce n'est pas le cas. Certes les rapports nationaux reflètent et même soulignent l'inégalité du développement de la recherche d'un pays à l'autre. Entre les résultats obtenus aux Etats-Unis d'un côté, et ceux sur lesquels peuvent s'appuyer le rapporteur australien ou son collègue finnois, la différence est sensible. "On the subject of pressure groups," écrit W. A. Townsley, "the first studies have yet to be made" (p. 9). De son côté Lola Krusius-Ahrenberg conclut son essai par cette remarque: "In spite of the influence they exercise, the pressure groups have thus far not been subject to scientific study and there is almost no literature dealing with their power and activities or with the question of their place in the political structure of Finland" (p. 58).

Mais la diversité des situations n'a pas constitué un obstacle majeur à l'entente nécessaire sur la manière d'aborder le sujet. Cela, on le doit très particulièrement à la méthode de travail adoptée par le rapporteur général. Henry W. Ehrmann, qui pouvait s'appuyer sur l'abondante littérature américaine sur le sujet et sur l'expérience pratique qu'il avait acquise lui-même par ses recherches sur la politique du patronat français, ${ }^{1}$ a su, en effet, fournir les définitions fondamentales, circonscrire le sujet et proposer un plan d'investigation acceptable qui dans l'essentiel a été suivi non pas seulement pour la rédaction des rapports nationaux, mais également lors des discussions qui ont eu lieu à Pittsburgh. Aussi n'est-il pas surprenant que les participants aient exprimé leur satisfaction du succès assez exceptionnel de cette conférence et leur désir de mettre leurs collègues moins privilégiés qu'eux au bénéfice des résultats qui avaient été ainsi obtenus. On ne saurait que les remercier. L'ouvrage,

${ }^{1}$ Henry W. Ehrmann, Organized Business in France, Princeton, N.J., 1957. 
en effet, constitue une excellente mise au point qui donne à chacun la possibilité de prendre conscience du travail accompli, des progrès à faire et des directions dans lesquelles s'engager.

\section{III}

Car il reste du chemin à parcourir, même aux Etats-Unis. Sur ce point, chacun s'accorde. En ce qui concerne l'identification des groupes de pression par exemple. Il suffit de se reporter au compte-rendu des débats de Pittsburgh pour se rendre compte de l'incertitude qui règne encore à ce sujet même parmi les spécialistes. Les définitions, pourtant, ne manquent pas, auxquelles les uns et les autres peuvent se référer; celle de David Truman par exemple, ou celle proposée par Jean Meynaud qui, comme le note Henry Ehrmann, sont assez proches l'une de l'autre. ${ }^{2}$ Mais le désaccord apparait au moment où l'on commence à discuter de leur application concrète, de manière à bien préciser ce qui distingue un groupe d'intérêts d'un parti politique, ou à définir les caractères de la pression.

C'est sur ce point précis, en effet, que les résultats sont les plus maigres. S'il est, à notre avis, possible d'identifier les groupes d'intérêts et même de marquer ce qui les distingue des partis politiques-quoique la limite ne soit pas toujours facile à fixer-il est beaucoup plus difficile de démontrer comment s'exerce la pression et surtout d'en fournir une preuve tangible. On peut bien établir des listes d'organisations diverses dont les statuts ou dont les déclarations des dirigeants révèlent les intentions. On peut même bâtir des schémas d'organisation, pour ne pas parler d'organigrammes, qui, mettant à jour le réseau des relations personnelles, dévoileraient du même coup les canaux par lesquels s'exercent les influences des groupements d'intérêts sur l'opinion, sur les partis, sur le législatif, sur l'administration ou sur le gouvernement. Mais le tableau le plus convaincant n'apporte rien de plus que des présomptions; de même la reconstitution des mécanismes par lesquels peut s'exercer la pression. Si l'on veut fournir la preuve qu'une pression a été réellement exercée, il faut disposer d'un dossier nourri de faits, et ces faits sont difficiles sinon parfois impossibles à établir. Sans doute est-il possible de raisonner par analogie et, grâce à une analyse poussée des structures

2 "Any group that, on the basis of one or more shared attitudes, makes certain claims upon other groups in the society for the establishment, maintenance or enhancement of forms of behavior that are implied by the shared attitudes" (Truman). "Associations of various juridical forms which upon the basis of common pools or attitudes endeavor to impose a certain number of positions and demands by all means at their disposal, but especially by pressure on the public authorities" (Meynaud). Ehrmann, op.cit., pp. 236-37. 
économiques, sociales et politiques, grâce à une reconstitution serrée des circonstances de tels évènements, d'établir un réseau d'hypothèses fort valables. Mais encore faut-il se rappeler qu'il ne s'agit là que d'hypothèses à vérifier. Or il n'est pas certain que la vérification donne les résultats qu'on attendait. Les recherches faites en histoire économique et sociale, les nombreuses études consacrées aux origines des guerres mondiales ont conduit à réviser certaines théories décidément trop simplistes dont une connaissance trop générale du déroulement des faits avait facilité l'élaboration. Ainsi en est-il, par exemple, de la théorie léniniste de l'impérialisme.

Aussi faut-il souhaiter que le nombre des monographies consacrées à des groupes de pression ou à l'histoire de groupements d'intérêts, d'organisations professionnelles ou d'associations de types divers ne cesse de croître. Sans se faire pourtant trop d'illusions. Les ouvrages de Jean Meynaud et surtout de Henry W. Ehrmann qui nous apportent de nombreuses informations intéressantes et inédites sur le patronat français, nous permettront-ils non seulement d'en suivre la politique, mais encore d'en mesurer l'influence au moment où se prennent certaines décisions politiques? Cela n'est pas certain. Une recherche que nous avons été amenée à faire sur un problème particulier, celui de la Sarre, nous a contraint tout d'abord à apprécier l'hermétisme des responsables, puis à modifier notre hypothèse de départ pour nous amener ensuite à constater qu'en fait la considération des intérêts politiques que présentait le rattachement économique de la Sarre à la France avait prévalu sur celle des intérêts économiques et que dans l'ensemble de la période considérée, les réactions du secteur-assez important-de l'industrie française touché par l'évolution de la question sarroise avaient été à la fois confuses et contradictoires.

Les industriels français, d'autre part, ont dû accepter finalement le Plan Schuman auquel ils avaient fait une opposition vigoureuse. Ce qui rappelle, comme le note Jean Meynaud, "que l'action des groupes de pression n'aboutit pas nécessairement au succès” (p. 269). Ce qui nous rend également attentifs au fait que les pressions des divers groupements d'intérêts ou des associations ne s'exercent pas toujours dans le même sens, que l'opinion, que les partis sont le plus souvent agités par des courants contradictoires et que de ce fait l'exécutif retrouve une relative liberté de décision et d'action. La multiplication des monographies, si désirable soit-elle, ne nous mène donc qu'à mi-chemin. Car c'est l'évaluation du rôle des groupes de pression dans la vie politique et de leur influence relative dans la conduite des affaires de la nation qui reste l'objectif final de toute étude les concernant. 


\section{IV}

Que dire alors de l'étude des groupes internationaux, si ce n'est qu'elle est à peine esquissée? Les historiens sans doute ont apporté déjà d'importantes contributions, très particulièrement pour la période antérieure à I9I4. On trouve également des renseignements utiles dans certaines des monographies qui ont été consacrées à des grandes compagnies internationales et à leur politique à l'égard de certains petits Etats ou de leur propre gouvernement. Mais il ne s'agit là, en fait, que de recherches assez fragmentaires et trop souvent marquées par le désir de démontrer. Aussi peut-on dire vraiment que l'essentiel reste à faire. Mais là de nouveau on se heurte au problème presque insoluble que pose l'accessibilité aux sources. Quelles sont en effet les grandes "corporations" qui sont disposées à ouvrir leurs archives au "political scientist"? Comment étudier de manière scientifique les relations des grandes compagnies pétrolières avec les Etats du Moyen-Orient? Les gouvernements sont-ils d'ailleurs plus disposés à fournir les documents? Encore faudrait-il qu'il y ait des documents ou que ces "documents" rendent compte des négociations qui bien souvent ne sont pas consignées dans un procès-verbal.

Les grandes "corporations" ne sont d'ailleurs pas les seuls groupements d'intérêts internationaux. Jean Meynaud, très justement, signale le vaste champ d'investigation qu'offrent les organisations internationales non gouvernementales. Il est bien probable, en effet, que la recherche puisse être poussée avec profit dans cette direction. Les organisations non gouvernementales représentent des intérêts extrêmement divers sans doute, mais identifiables. Elles peuvent avoir des objectifs plus ou moins précis, mais elles n'en cherchent pas moins à exercer une influence selon des méthodes et des techniques dont il n'est pas sans intérêt de prendre connaissance. Il est très certain qu'un examen attentif de leur action, celle par exemple des grandes organisations syndicales internationales, à l'intérieur des Etats ou au sein des organisations internationales permettrait de combler certaines lacunes dans notre connaissance et dans nos méthodes d'analyse des relations internationales. 